\title{
Memory Functioning in Psychopathology
}

\author{
Roseli S. Wedemann ${ }^{1}$, Raul Donangelo ${ }^{2}$, \\ Luís Alfredo V. de Carvalho ${ }^{3}$, and Isa H. Martins ${ }^{1}$ \\ 1 Instituto de Matemática e Estatística \\ Universidade do Estado do Rio de Janeiro \\ Rua S. Francisco Xavier, 524 \\ 20550-013 Rio de Janeiro, RJ, Brazil \\ $\{$ roseli, isa\}@ime.uerj.br \\ 2 Instituto de Física \\ Universidade Federal do Rio de Janeiro \\ Caixa Postal 68528 \\ 21945-970 Rio de Janeiro, RJ, Brazil \\ donangel@if.ufrj.br \\ 3 Programa de Sistemas, COPPE - LUPA, UniCarioca \\ Universidade Federal do Rio de Janeiro \\ Caixa Postal 68511 \\ 21945-970 Rio de Janeiro, RJ, Brazil \\ alfredo@cos.ufrj.br
}

\begin{abstract}
The mental pathology known as neurosis, its aetiology, and the development of symptoms are described in terms of their relation to memory function. We propose, based on a neural network model, that neurotic behavior may be understood as an associative memory process in the brain, and that the linguistic, symbolic associative process involved in psychoanalytic working-through can be mapped onto a corresponding process of reconfiguration of the neural network. The model is illustrated through a computer simulation implementation. We relate the sensitivity to temperature and the adaptive capabilities of our model, with the sensitivity of cortical map modulation to the catecholamines (norepinephrine and dopamine). The signal-to-noise ratio regulated by these substances influence thought associativity, suggesting a continuum from psychotic functioning through to normal and neurotic behavior and creativity.
\end{abstract}

\section{Introduction}

We propose a schematic functional model for some concepts associated with neurotic mental processes as described by Sigmund Freud and further developed on by Jacques Lacan [1-7]. Our description is based on the current view that the brain is a cognitive system composed of neurons, interconnected by a network of synapses, that cooperate locally among themselves to process information in a distributed fashion. Mental states thus appear as the result of the global cooperation of the distributed neural cell activity in the brain. We also consider that the emergence of a global state of the neural network of the brain generates a bodily response which we will call an act. 
Based on the view of the brain as a parallel and distributed processing system $[8,9]$, we assume that human memory is encoded in the architecture of the neural net of the brain. By this we mean that we record information by reconfiguring the topology of our neural net, i.e. the set of active neurons and synapses that interconnect them to each other, along with the intensities and durations of these connections [10]. We will often refer to this reconfiguration process as learning.

Once a memory trace has been stored, whenever a stimulus excites the neural net to a certain energy state $S$, the net will stabilize into a local minimum energy state $S_{m}$, in which the circuit corresponding to the stored memory trace most similar to the stimulus becomes active, and, as a result generating the respective output. This is referred to as an associative memory mechanism $[11,8]$.

Since there is no clear consensus on the relevance of quantum effects in the macroscopic phenomena that underly molecular and cellular activity in the brain, we therefore take a classical approximation of these phenomena. In particular, we disregard the possibility of the occurrence of nondeterministic events in brain activity caused by quantum effects. We thus attribute any unpredictability in mental behavior to the sensitivity of the non-linear, complex neural networks to initial states and to internal and external parameters, which cannot be determined exactly.

Finally, we assume that each brain state (global state of the neural network) represents only one mental state. That is equivalent to affirming, in linguistic terms, that each symbol is associated to only one significance (meaning), so that we have a one-to-one functional mapping. We suggest that the symbol is represented physiologically by a minimal energy state of the neural net configuration, which encodes the memorized symbolic information.

The remainder of the paper is organized as follows. In the next section, we introduce concepts associated with neurotic phenomena as described by Freud, which we model in sections 3 and 4 . In section 5 we describe the simulation experiments and their results, and draw our conclusions and perspectives for further work.

\section{Basic Concepts Associated with Neuroses}

As a result of the assumptions we have made in the introduction, each repressed or traumatic memory trace stored in our brain is associated, as other non-traumatic memories, to one of the possible minimum energy states of our biological neural net.

It is one of the early findings of psychoanalytic research regarding the transference neuroses, that traumatic and repressed memories are knowledge which is present in the subject but which is inaccessible to him, i.e. momentarily or permanently inaccessible to the subject's conscience. It is therefore considered unconscious knowledge $[12,13,1]$. They arise from events which give the mind a stimulus too powerful to be dealt with in the normal way, and thus result in permanent disturbances. 
Freud observed that his neurotic patients systematically repeated symptoms in the form of ideas and impulses. This tendency to systematically repeat symptoms was called by Freud a compulsion to repeat [2]. He related the compulsion to repeat to repressed or traumatic memory traces. The original cause of the repression is given by a conflict associated with libidinal fixation and frustration. The neurosis installs itself in the form of " a contention between wishful impulses, or, ... a psychical conflict. One part of the personality champions certain wishes while another part opposes them and fends them off." [1].

The result of clinical experience in psycho-analysis since late nineteenth century has revealed that patients with strong neurotic symptoms have been able to obtain relief and cure of painful symptoms through a mechanism called workingthrough [3]. The procedure aims at developing knowledge regarding the causes of symptoms, by accessing unconscious memories. Psycho-analysis aims not only at constructing conscious knowledge of the repressed, unconscious material, but beyond that and most importantly, at understanding and changing the way in which the analysand obtains satisfaction $[2,6]$. For this purpose, it is fundamental that the analyst positions himself in the sessions in a manner that compels the analysand to a new outcome. Lacan emphasizes the creative nature of transference [14]. A mere repetition, such as that which takes place in our everyday life, simply reinforces the traumas and repressions.

\section{Functional Model for the Neuroses}

We propose that the neuroses manifest themselves as an associative memory process. An associative memory is a mechanism where the network returns a given stored pattern when it is shown another input pattern sufficiently similar to the stored one $[11,8]$. Although neural networks have been proposed to help diagnose neuroses and schizophrenia [15], we are not aware of any previous effort to model neuroses with a distributed processing, neural network approach.

We propose in our model that the compulsion to repeat neurotic symptoms can be described by supposing that a neurotic symptom is acted when the subject is presented with a stimulus which resembles, at least partially, a repressed or traumatic memory trace. The stimulus causes a stabilization of the neural net onto a minimal energy state corresponding to the original memory trace, which in turn generates a neurotic response (an act).

In neurotic behavior associated with a stimulus, the act is not a result of the stimulus as a new situation but a response to the original repressed memory trace. The original repression can be accounted for by a mechanism which inhibits the formation of certain synaptic connections. The inhibition may be externally imposed, for example by cultural stimulation or the relation with the parents, and internalized so that the subject inhibitively stimulates the regions associated with the memory traces, not allowing the establishment of certain synaptic connections.

We thus map the linguistic, symbolic associative process involved in psychoanalytic working-through into a corresponding process of reinforcing synapses 
among memory traces in the brain. These connections should involve declarative memory, leading to at least partial transformation of the repressed memory to consciousness. This has a relation to the importance of language in psychoanalytic sessions and the idea that unconscious memories are those that cannot be expressed.

We propose that as the analysand symbolically elaborates the manifestations of unconscious material and creates new editions of old conflicts through transference in psychoanalytic sessions, he is reconfiguring the topology of the neural net in his brain by actually creating new connections and reinforcing older ones, among the subnetworks that store the repressed memory traces. The network topology which results from this reconfiguration process will stabilize onto new energy minima associated with new conscious or unconscious responses or acts.

In our model, it is clear why repetition in psychoanalysis is specially important. Neuroscience has established that memory traces are established by repeatedly reinforcing, through stimulation, the appropriate synaptic connections. This is exactly the learning process in a neural network and accounts for the long durations of psychoanalytic processes. Much time may be needed to overcome resistances in order to access and interpret repressed material, and even more to repeat and reconfigure the net in a learning process.

Our model differentiates, from the physiological point of view, psychoanalytic working-through from the neurotransmitting drug therapy, characteristic of psychiatric treatment. Drugs change network response by having a more global effect over the net. This global effect cannot account for the selective fine-tuning process achieved by reconfiguring individual synapses through psychoanalytic working -through.

\section{Computational Model}

Memory functioning is modeled by a Boltzmann machine [11,16] We consider $N$ nodes, which are connected symmetrically by weights $w_{i j}=w_{j i}$. The states of the units $S_{i}$, take output values in $\{-1,1\}$. Because of the symmetry of the connections, there is an energy functional

$$
H\left(\left\{S_{i}\right\}\right)=-\frac{1}{2} \sum_{i j} w_{i j} S_{i} S_{j}
$$

which allows us to define the Boltzmann distribution function for network states

$$
P\left(\left\{S_{i}\right\}\right)=\exp \left[-\frac{H\left(\left\{S_{i}\right\}\right)}{T}\right] / \sum_{\left\{S_{i}\right\}} \exp \left[-\frac{H\left(\left\{S_{i}\right\}\right)}{T}\right],
$$

where $T$ is the network temperature parameter. Pattern retrieval on the net is achieved by a standard simulated annealing process, in which the network temperature $T$ is gradually lowered by a factor $\alpha$.

In our simulations, initially, we take random connection weights $w_{i j}$. We also consider that the network is divided into two weakly linked subsets, representing 
the conscious and unconscious parts of the memory. This is done by multiplying the connections between the two subsets by a number less than one.

Once the network is initialized, we find the stored patterns by presenting many random patterns to the Boltzmann machine, with an annealing schedule $\alpha$ that permits stabilizing onto the many local minimum states of the network energy function. These initially stored patterns, associated as they are to two weakly linked subnetworks, represent the neurotic memory states. One verifies that when initializing the "conscious" nodes of the memory into one of these states and the "unconscious" nodes on a random configuration, the net rapidly evolves into one of the neurotic memory states. In [17], the authors propose a neurocomputational model to describe how the original memory traces are formed in cortical maps.

In order to simulate the working-through process, one should stimulate the net by means of a change in a randomly chosen node $n_{i}$ belonging to the "unconscious" section of a neurotic memory pattern. This stimulus is then presented to the network and, if the Boltzmann machine retrieves a pattern with conscious configuration different than that of the neurotic pattern, we interpret this as a new conscious association, and enhance all weights from $n_{i}$ to the changed nodes in the conscious cluster. The increment values are given by

$$
\Delta w_{i j}=\beta * S_{i} * S_{j} * w_{\max },
$$

where $\beta$ is the learning parameter chosen in $(0,1)$, and $w_{\max }$ the maximum absolute initial synaptic strengths. We note that new knowledge is learned only when the stimulus from the analyst is not similar to the neurotic memory trace.

This procedure must be repeated for various reinforcement iterations in an adaptive learning procedure, and also repeat each set of reinforcement iterations for various initial annealing temperature values. The new set of synaptic weights will define a new network configuration.

\section{Simulation Model Illustration}

For a network with $N=32$ nodes such that $N_{u n c}=16$ of them belong to the unconscious subset, the model depends on the parameters listed in table 1 , which also gives the corresponding values for our simulation experiment. The memory configurations before working-through are shown in table 2. After the learning process, one obtains a new set of patterns shown in table 3 . In both tables, minimum energy values are listed in the last column. Many repetitions, are needed in the learning process for the observation of new network states.

Table 4 shows the associativity capability of the network before workingthrough, i.e. of a neurotic network, as a function of initial annealing temperature, by presenting a single random pattern to the Boltzmann machine and varying the temperature. The second column lists the energy value associated with the pattern onto which the machine stabilizes for each temperature. With a small annealing schedule, we see in table 4 that as temperature is varied in the model, new memory configurations are attainable from a given initial pattern. In our 
Table 1. Parameter set for illustrative simulation experiment.

\begin{tabular}{|l|l|}
\hline$N$ & 32 \\
number of nodes in each subset, $N_{u n c}$ & 16 \\
initial temperature for finding global minimum with simulated annealing & 1.0 \\
initial temperature for seeking local minima with simulated annealing & 0.05 \\
lowest temperature for simulated annealing & 0.001 \\
$\alpha$ for finding global minimum & 0.95 \\
$\alpha$ for finding local minima & 0.50 \\
number of iterations per temperature in simulated annealing & 20 \\
number of trial patterns for initial configuration pattern detection & 10000 \\
parameter for initializing intercluster synapses & 0.5 \\
$\beta$ & 0.3 \\
number of iterations for reinforcement learning & 1000 \\
\hline
\end{tabular}

initial experiments, some simulations required a temperature increase to allow association of stimulus from the analyst from a neurotic state to a new conscious configuration.

In neural network modeling, temperature is inspired from the fact that real neurons fire with variable strength, and there are delays in synapses, random fluctuations from the release of neurotransmitters, and so on. These are effects that we can loosely think of as noise $[11,8,10]$. So temperature in Boltzmann machines controls noise. In our model, temperature, i.e. noise, allows associativity among memory configurations, lowering synaptic inhibition, in an analogy with the idea that freely talking analytic sessions, and stimulation from the analyst lower resistances and allow greater associativity.

It is also possible to relate our simulation experiments with the model described in [17], where the signal-to-noise ratio at neuronal level, induced by the catecholamines (dopamine, norepinephrine, etc.), alter the way the brain performs associations of ideas. An increase of dopamine (hyperdopaminergy) promotes an enhancement of signal and therefore of the signal-to-noise ratio. Since noise is responsible for more free association of ideas, its reduction weakens associativity resulting in fixation to certain ideas. On the other hand, a decrease in dopamine increases noise which permits a broader association of ideas. Certainly, excessive noise may disorganize thought by permitting the association of distantly correlated ideas and breaking the logic of thought. This suggests a phenomenologic continuum from low signal-to-noise ratio (low dopamine level) corresponding to logically disorganized thought, passing through a slightly low dopamine level associated with creative thought and arriving at high signal-tonoise ratios, responsible for fixations to ideas and delusions.

We can thus relate temperature and noise in our Boltzmann machine to associativity of thought as in [17]. Very high temperatures, allow the production of logically disorganized thought because they allow associations of distant, usually uncorrelated ideas as in the low signal-to-noise ratio characteristic of low dopamine levels. However, at low temperatures, the Boltzmann machine inhibits 
Table 2. Memorized patterns for initial network configuration.

\begin{tabular}{|lllllllllllllllllllllllllllllllll|}
\hline & \multicolumn{10}{c|}{ Conscious } \\
\hline 0 & 1 & 1 & 0 & 1 & 1 & 1 & 1 & 0 & 1 & 0 & 0 & 1 & 0 & 1 & 1 & 0 & 1 & 1 & 0 & 0 & 1 & 0 & 1 & 1 & 0 & 0 & 0 & 1 & 1 & 0 & 0 & -216.2 \\
0 & 1 & 1 & 1 & 1 & 1 & 1 & 1 & 0 & 1 & 1 & 0 & 1 & 0 & 1 & 1 & 0 & 0 & 1 & 0 & 1 & 1 & 0 & 1 & 1 & 0 & 0 & 0 & 1 & 1 & 1 & 0 & -208.2 \\
0 & 1 & 1 & 0 & 1 & 1 & 1 & 1 & 0 & 1 & 0 & 0 & 1 & 0 & 1 & 1 & 0 & 1 & 1 & 0 & 1 & 1 & 0 & 1 & 0 & 0 & 0 & 0 & 1 & 1 & 1 & 0 & -215.0 \\
0 & 1 & 1 & 0 & 1 & 1 & 1 & 1 & 0 & 1 & 0 & 0 & 1 & 0 & 1 & 1 & 0 & 0 & 1 & 0 & 0 & 1 & 0 & 1 & 1 & 0 & 0 & 0 & 1 & 1 & 1 & 0 & -215.6 \\
0 & 1 & 1 & 0 & 1 & 1 & 1 & 1 & 0 & 1 & 0 & 0 & 1 & 0 & 1 & 1 & 0 & 0 & 1 & 0 & 0 & 1 & 0 & 1 & 1 & 0 & 0 & 0 & 1 & 1 & 0 & 1 & -215.9 \\
0 & 1 & 1 & 0 & 1 & 1 & 1 & 0 & 0 & 0 & 1 & 0 & 1 & 0 & 1 & 1 & 0 & 0 & 0 & 0 & 0 & 0 & 1 & 0 & 1 & 1 & 0 & 1 & 0 & 0 & 0 & 1 & -167.8 \\
1 & 1 & 1 & 0 & 1 & 1 & 1 & 1 & 0 & 1 & 0 & 0 & 1 & 0 & 1 & 1 & 0 & 1 & 0 & 0 & 1 & 1 & 0 & 0 & 1 & 0 & 0 & 0 & 1 & 1 & 0 & 0 & -208.2 \\
0 & 1 & 1 & 1 & 1 & 1 & 1 & 1 & 0 & 1 & 1 & 0 & 1 & 0 & 1 & 1 & 0 & 1 & 1 & 1 & 1 & 1 & 0 & 0 & 1 & 0 & 1 & 0 & 1 & 1 & 0 & 0 & -200.9 \\
0 & 1 & 1 & 1 & 1 & 1 & 1 & 0 & 0 & 0 & 1 & 0 & 1 & 0 & 1 & 1 & 1 & 0 & 1 & 0 & 0 & 0 & 1 & 0 & 1 & 0 & 0 & 1 & 0 & 0 & 0 & 0 & -169.1 \\
0 & 1 & 1 & 1 & 1 & 1 & 1 & 0 & 0 & 0 & 1 & 0 & 1 & 0 & 1 & 1 & 1 & 0 & 1 & 0 & 0 & 0 & 1 & 0 & 1 & 1 & 0 & 1 & 0 & 0 & 0 & 1 & -171.4 \\
0 & 1 & 1 & 1 & 1 & 1 & 1 & 1 & 0 & 1 & 1 & 0 & 1 & 0 & 1 & 1 & 0 & 0 & 1 & 0 & 0 & 1 & 0 & 0 & 1 & 0 & 0 & 1 & 1 & 1 & 0 & 0 & -198.1 \\
1 & 0 & 0 & 1 & 0 & 1 & 1 & 0 & 1 & 0 & 1 & 0 & 0 & 0 & 1 & 1 & 0 & 1 & 0 & 1 & 1 & 0 & 1 & 0 & 0 & 1 & 0 & 1 & 0 & 0 & 0 & 0 & -128.9 \\
1 & 0 & 0 & 1 & 0 & 1 & 1 & 0 & 1 & 0 & 1 & 0 & 1 & 0 & 1 & 1 & 1 & 0 & 1 & 0 & 1 & 0 & 1 & 0 & 0 & 0 & 0 & 1 & 0 & 0 & 1 & 0 & -133.9 \\
0 & 1 & 0 & 1 & 0 & 1 & 1 & 0 & 1 & 0 & 1 & 1 & 0 & 1 & 0 & 1 & 1 & 1 & 1 & 1 & 0 & 1 & 1 & 0 & 0 & 0 & 0 & 0 & 0 & 0 & 0 & 0 & -105.0 \\
\hline
\end{tabular}

the associative process becoming fixated in a local miminum of the energy function, equivalent to a hyperdopaminergic state characteristic of delusions and inflexible thought.

It is also known that norepinephrine levels control synaptic learning. Low or high norepinephrine levels corresponding to much or little noise respectively, inhibit the establishment of synapses and learning. Psychoanalysis promotes new thought associations and learning, and this cannot be carried through if norepinephrine and dopamine levels are too high or too low. We suggest that psychoanalytic working-through explores the neurophysiologic potentialities of the subject, inducing network reconfiguration. When these potentialities are limited by chemical alterations, such as in strong psychotic cases, working-through should be limited or even impossible.

The model is in agreement with psychoanalytic experience that workingthrough is a slow process, where the individual slowly elaborates knowledge by re-associating weakly connected memory traces and new experiences. This selfreconfiguration process, which we represent in the model by a change in network connectivity, will correspond to new outcomes in the subjects life history. Repetition is an important component of this process and our model illustrates this by the repetitive adaptive reinforcement learning involved in the simulation of working-through. Although biologically plausible and in accordance with aspects of clinical experience described by psycho-analysis, the model is very schematic and far from explaining the complexities of mental processes. Although still in lack of experimental verification, it seams to be a good metaphorical view of the basic concepts of neurotic behavior described by Freud, to which we have referred. 
We are now proceeding to systematically study the parameter dependency of the model. If possible, we will try an interpretation of these parameter dependencies as associated to memory functioning in the brain and psychic apparatus functioning.

\section{Acknowledgements}

We are grateful for fruitful discussions regarding basic concepts of psychoanalytic theory, with the psychoanalyst Professor Angela Bernardes of the Escola Brasileira de Psicanálise of Rio de Janeiro and the Department of Psychology of the Federal Fluminense University, psychoanalyst Dr. Nicolau Maluf and Eduardo Aguilar, both graduate students in neuroscience of the Federal University of Rio de Janeiro. We also thank the members of the Escola Brasileira de Psicanálise of Rio de Janeiro for their warm reception of the author R.S. Wedemann in some of their seminars. The initial discussions regarding language and logic of the unconscious with Professor Ricardo Kubrusly of the Institute of Mathematics of the Federal University of Rio de Janeiro were also enlightening. We acknowledge partial financial support from the Conselho Nacional de Desenvolvimento Científico e Tecnológico ( $\mathrm{CNPq})$.

Table 3. Memorized patterns after the simulated working-through process.

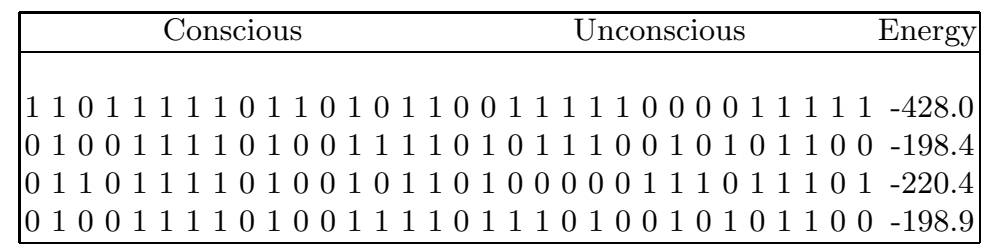

\section{References}

1. Freud, S.: Introductory Lectures on Psycho-Analysis. Standard Edition . W. W. Norton and Company, 1966. First German edition in 1917.

2. Freud, S.: Beyond the Pleasure Principle. Standard Edition . The Hogarth Press, London, 1974. First German edition in 1920.

3. Freud, S.: Remembering, Repeating and Working-Through. Standard Edition. Volume XII, The Hogarth Press, London, 1953-74. First German edition in 1914.

4. Freud, S.: The Ego and the Id. Standard Edition . W. W. Norton and Company, 1960. First German edition in 1923.

5. Lacan, J.: O Seminário, Livro 2: O Eu na Teoria de Freud e na Técnica da Psicanálise. Jorge Zahar Editor, Brazil, 1985.

6. Bernardes, A.C.: Elaboration of Knowledge in Psychoanalysis, a Treatment of the Impossible. Doctoral thesis. Programa de Pós-graduação em Teoria Psicanalítica, UFRJ, Rio de Janeiro, Brazil, 2000 (in Portuguese). 
7. Forrester, J.: The Seductions of Psychoanalysis, Freud, Lacan and Derrida. Cambridge University Press, 1990.

8. Rumelhart, D.E., McCleland, J.L. (ed.): Parallel Distributed Processing: Explorations in the Microstructure of Cognition. 2 Volumes . The MIT Press, Cambridge, MA, 1986.

9. Varela, F.J., Thompson, E., Rosch, E.: The Embodied Mind. The MIT Press, Cambridge, MA, 1997.

10. Kandel, E.R., Schwartz, J.H., Jessel, T.M.: Principles of Neural Science. MacGraw Hill, 2000.

11. Hertz, J.A., Krogh, A., Palmer, R.G. (ed.): Introduction to the Theory of Neural Computation. Lecture Notes, Vol. I, Santa Fe Institute, Studies in the Science of Complexity, 1991.

12. Janet, P.: Les actes inconscients et la memoire. Rev. Philosoph., 13, 238. (319), 1888.

13. Breuer, J., Freud, S.: On the Psychical Mechanism of Hysterical Phenomena: Preliminary Communication. Collected Papers 1, 24; Standard Edition, London 192450. First German edition in 1893.

14. Lacan, J.: O Seminário, Livro 8: A Transferência. Jorge Zahar Editor, Brazil, 1992.

15. Zou Y.Z., Shen, Y.C., Shu, L.A. et al. : Artificial neural network to assist psychiatric diagnosis. Brit. J. Psychiat. 169: (1) 64-67 Jul. 1996.

16. Barbosa, W.C.: Massively Parallel Models of Computation. Ellis Horwood Limited, 1993.

17. Mendes, D.Q., Carvalho, L.A.V.: Creativity and Delusions: The Dopaminergic Modulation of Cortical Maps. Submitted to the Journal of Theoretical Medicine in 2001. 
Table 4. Associativity capability of the neurotic network.

\begin{tabular}{|c|l|}
\hline Temperature & Energy \\
\hline 0.001 & -208.2 \\
0.04 & -216.2 \\
0.08 & -216.2 \\
0.10 & -215.0 \\
0.14 & -216.2 \\
0.18 & -215.6 \\
0.20 & -216.2 \\
0.24 & -215.0 \\
0.28 & -216.2 \\
0.30 & -215.9 \\
0.34 & -215.9 \\
0.38 & -216.2 \\
0.40 & -216.2 \\
0.44 & -216.2 \\
0.48 & -215.9 \\
0.50 & -215.9 \\
0.54 & -216.2 \\
0.58 & -216.2 \\
0.60 & -216.2 \\
0.64 & -216.2 \\
0.68 & -216.2 \\
0.70 & -216.2 \\
0.74 & -215.9 \\
0.78 & -215.0 \\
0.80 & -216.2 \\
0.84 & -216.2 \\
0.88 & -216.2 \\
0.90 & -216.2 \\
0.94 & -215.6 \\
0.98 & -215.6 \\
0.99 & -215.9 \\
\hline
\end{tabular}

